\title{
Congenital Adrenocortical Tumor in an Asymptomatic Neonate with Positive Newborn Screen for Congenital Adrenal Hyperplasia: A Case Report
}

\author{
Phuong Thao Hoang*, Eric Tsay, John Mace and Eba Hathout
}

\begin{abstract}
Division of Pediatric Endocrinology and Diabetes, Loma Linda University Children's Hospital and Loma Linda University School of Medicine, Loma Linda, CA 92354, USA
\end{abstract}

\begin{abstract}
Adrenocortical tumors (ACTs) are rare in childhood, particularly in the neonatal period. ACTs in children usually secrete hormones that can cause virilization, precocious puberty, or Cushing syndrome. However, in many cases the diagnosis is delayed because of the generally healthy appearance of the child and the lack of a palpable abdominal mass. ACTs can also be misdiagnosed as congenital adrenal hyperplasia $(\mathrm{CAH})$ especially if 17-hydroxyprogesterone (17-OHP) is elevated.

We describe a case of neonatal ACT diagnosed in an asymptomatic newborn with positive/elevated 17OHP on newborn screening. On examination there were no signs of virilization, Cushing syndrome, hemihypertrophy, or hypertension, and there was no palpable abdominal mass. While awaiting confirmatory results of an elevated 17-OHP level, an abnormally low adrenocorticotropic hormone level was noted. Abdominal ultrasound, obtained to evaluate the adrenal glands, showed a right adrenal mass with peripheral calcification. MRI revealed a $3.6 \times 3.5 \times 3.4 \mathrm{~cm}$ heterogeneously enhancing ovoid mass in the right adrenal gland. Diagnosis of an ACT was also confirmed by laboratory data, and histopathology of the tumor. Surgical resection was safely performed with perioperative steroid replacement. Genetic analysis of CYP21A2 and CYP11B1 genes was negative. A germ-line point mutation in the TP53 region was discovered, suggesting Li-Fraumeni syndrome.
\end{abstract}

ACTs should be considered in the differential diagnosis of neonates with elevated 17-OHP detected by newborn screening for $\mathrm{CAH}$. Adrenal ultrasound is recommended in cases of significantly abnormal $\mathrm{CAH}$ screen results to rule out or treat adrenal neoplasms.

Keywords: Adrenocortical tumor, newborn screening, 17-hydroxyprogesterone, congenital adrenal hyperplasia, Li-Fraumeni syndrome.

\section{INTRODUCTION}

Adrenocortical tumors (ACTs) are extremely rare in childhood, particularly in the neonatal period [1]. ACTs in children usually secrete hormones that can cause virilization, precocious puberty, or Cushing syndrome [4,5]. Congenital adrenal hyperplasia $(\mathrm{CAH})$ presents with virilization, precocious puberty, and elevated androgen hormones. Diagnosis of ACTs is often delayed because of the patient's generally healthy appearance and the absence of a palpable abdominal mass [5]. ACTs can be misdiagnosed as $\mathrm{CAH}$, especially if 17-hydroxyprogesterone $(17-\mathrm{OHP})$ is elevated.

\section{CASE DESCRIPTION}

The patient's parents provided informed consent and the report followed the guidelines of the

*Address correspondence to Phuong Thao Hoang; Division of Pediatric Endocrinology and Diabetes, Loma Linda University Children's Hospital and Loma Linda University School of Medicine; 11175 Campus Street, CP A1120, Loma Linda, CA 92354; Tel: 909558-4773; Fax: 909-558 -0479; Email: PTHoang@llu.edu institutional review board of Loma Linda University School of Medicine.

A 5-day-old female was admitted to the hospital for diagnostic evaluation and treatment of suspected $\mathrm{CAH}$ due to extremely elevated 17 OHP on the newborn screen. A result of 509.2 $\mathrm{nmol} / \mathrm{L}(16,805.3 \mathrm{ng} / \mathrm{dL}$ ) was observed (cutoff levels $=70 \mathrm{nmol} / \mathrm{L}$ and $2,310.2 \mathrm{ng} / \mathrm{dL}$ ). The patient was born vaginally at full term after an uncomplicated pregnancy and delivery. Family history was negative for consanguinity, $\mathrm{CAH}$, ACTs, and other cancers. The patient was feeding well and hemodynamically stable. Examination disclosed no signs of virilization, Cushing syndrome, hemi-hypertrophy, hypertension, or palpable abdominal masses.

An abnormally low random adrenocorticotropic hormone $(\mathrm{ACTH})$ level was noted while awaiting confirmatory results of circulating 17-OHP. Abdominal ultrasound, obtained to evaluate the adrenal glands, revealed a right adrenal mass with peripheral calcification (Figure 1). MRI of the 


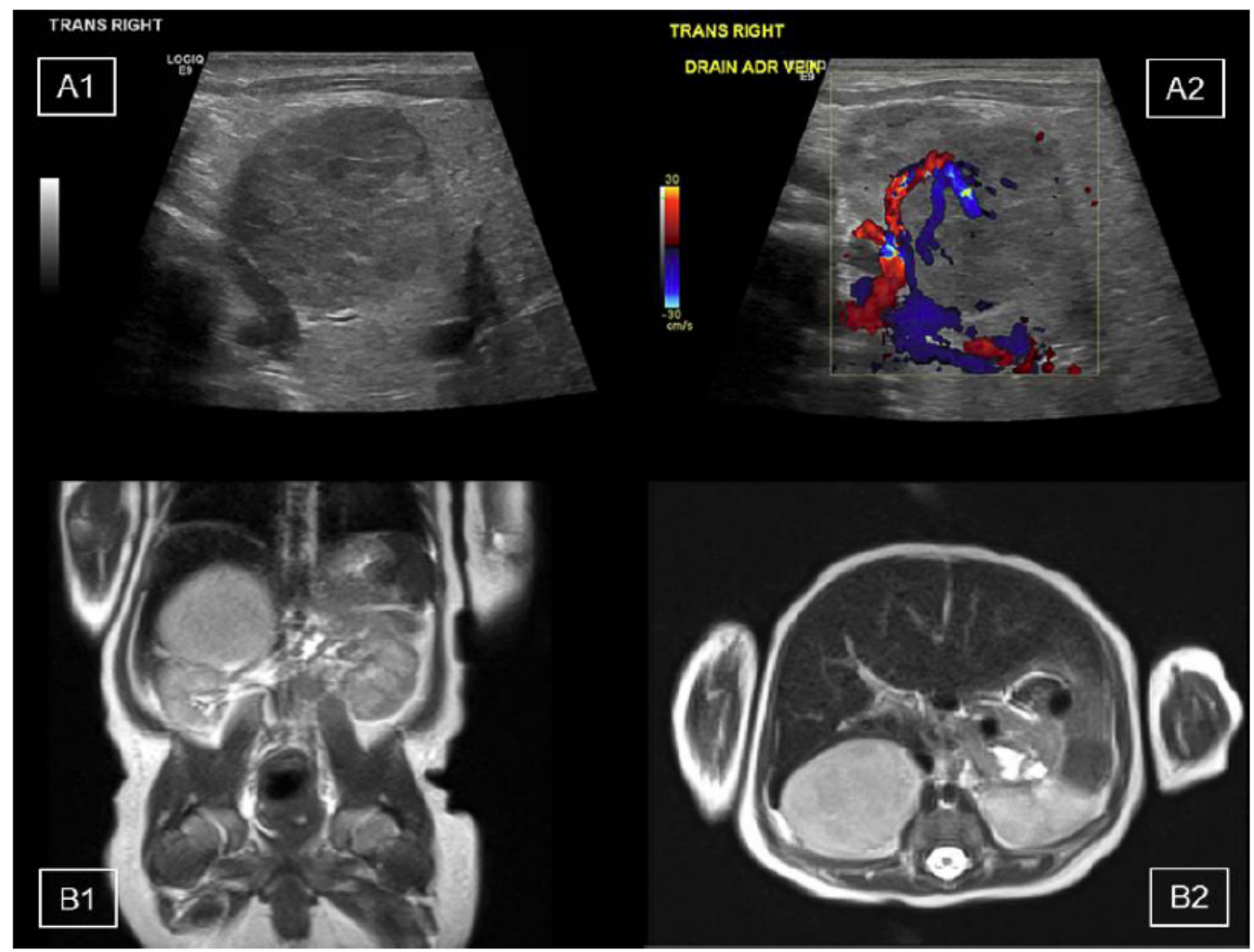

Figure 1: Abdominal ultrasound shows a heterogeneous adrenal mass (A1) with prominent feeding vessels (A2); Axial (B1) and coronal (B2) MRI T2 images show an iso-dense to hyper-intense enhancing mass arising from right adrenal fossa.

abdomen with and without contrast revealed a 3.6 x $3.5 \times 3.4 \mathrm{~cm}$ heterogeneously enhancing ovoid mass in the right adrenal gland (Figure 1). Imaging disclosed no pulmonary, hepatic, peritoneal, lymphatic, or skeletal metastases.

Further investigation confirmed elevated 17-OHP, $136.35 \mathrm{nmol} / \mathrm{L} \quad(4,500 \mathrm{ng} / \mathrm{dL})$ (normal: <19.1 $\mathrm{nmol} / \mathrm{L}$ or $<630 \quad \mathrm{ng} / \mathrm{dL}) ; \quad$ 17-alphahydroxypregnenolone, $647.2 \mathrm{nmol} / \mathrm{L} \quad(21,500$ $\mathrm{ng} / \mathrm{dL}$ ) (normal: $0.3-24.9 \mathrm{nmol} / \mathrm{L}$ or $10-829 \mathrm{ng} / \mathrm{dL}$ ); 11-deoxycortisol, $36.7 \mathrm{nmol} / \mathrm{L} \quad(1,270 \mathrm{ng} / \mathrm{dL})$ (normal: $0.38-4.2 \mathrm{nmol} / \mathrm{L}$ or $13-147 \mathrm{ng} / \mathrm{dL}$ ); dehydroepiandrosterone (DHEA), $65.6 \mathrm{nmol} / \mathrm{L}$ $(1,890 \mathrm{ng} / \mathrm{dL})$ (normal: $2.3-43.4 \mathrm{nmol} / \mathrm{L}$ or $65-1250$ $\mathrm{ng} / \mathrm{dL})$; and total testosterone, $4.9 \mathrm{nmol} / \mathrm{mL}$ (144 $\mathrm{ng} / \mathrm{dL}$ ) (normal: $0.2-2.8 \mathrm{nmol} / \mathrm{L}$ or $6-82 \mathrm{ng} / \mathrm{dL}$ ). Stimulated 17-OHP level was increased to 209.37 $\mathrm{nmol} / \mathrm{L}(6,910 \mathrm{ng} / \mathrm{dL})$ (normal: $<19.1 \mathrm{nmol} / \mathrm{L}$ or $<630 \mathrm{ng} / \mathrm{dL}$ ) after cortrosyn stimulation test. Abnormal results were found following low-dose dexamethasone suppression test, with an elevated cortisol level of 2,594.4 nmol/L (94 $\mathrm{mcg} / \mathrm{dL}$ ) (normal: $<49.7 \mathrm{nmol} / \mathrm{L}$ or $<1.8 \mathrm{mcg} / \mathrm{dL}$ ). In a 24-hour urine collection, free cortisol was elevated to $209.8 \mathrm{nmol} / \mathrm{d}$ and $248.4 \mathrm{nmol} / \mathrm{m}^{2}$ body surface area/d $\left(76 \mathrm{mcg} /\right.$ day and $90 \mathrm{mcg} / \mathrm{m}^{2} /$ day $)$ (normal: $69-207 \mathrm{nmol} / \mathrm{m}^{2} /$ day or $25-75 \mathrm{mcg} / \mathrm{m}^{2}$ per day). Renal function, serum glucose, urine homovanillic acid, urine vanillylmandelic acid, plasma metanephrines, aldosterone, and plasma renin activity were all normal.

After perioperative steroid replacement, a right adrenalectomy was successfully performed. A round solid tumor measuring $5.5 \times 4.5 \times 3.5 \mathrm{~cm}$ with a smooth capsule/peritoneal surface was completely removed together with the right adrenal gland (Figure 2). The tumor weighed $40 \mathrm{~g}$. No metastases were noticed during the operation.

Tumor histopathology confirmed an adrenocortical neoplasm with features consistent for malignancy. This ACT contained cells with eosinophilic cytoplasm and heterogeneous nuclei with pleomorphism (Figure 2). High rates of mitosis and nuclear atypia were observed. Features favoring a benign prognosis included the weight $<50 \mathrm{~g}$ and lack of vascular and capsular invasion. Features concerning for malignancy included the relative high mitotic rate, occasional atypical mitoses, multifocal necrosis, lack of a clear cell component, incomplete intra-adrenal encapsulation, and a tendency toward sheet-like 


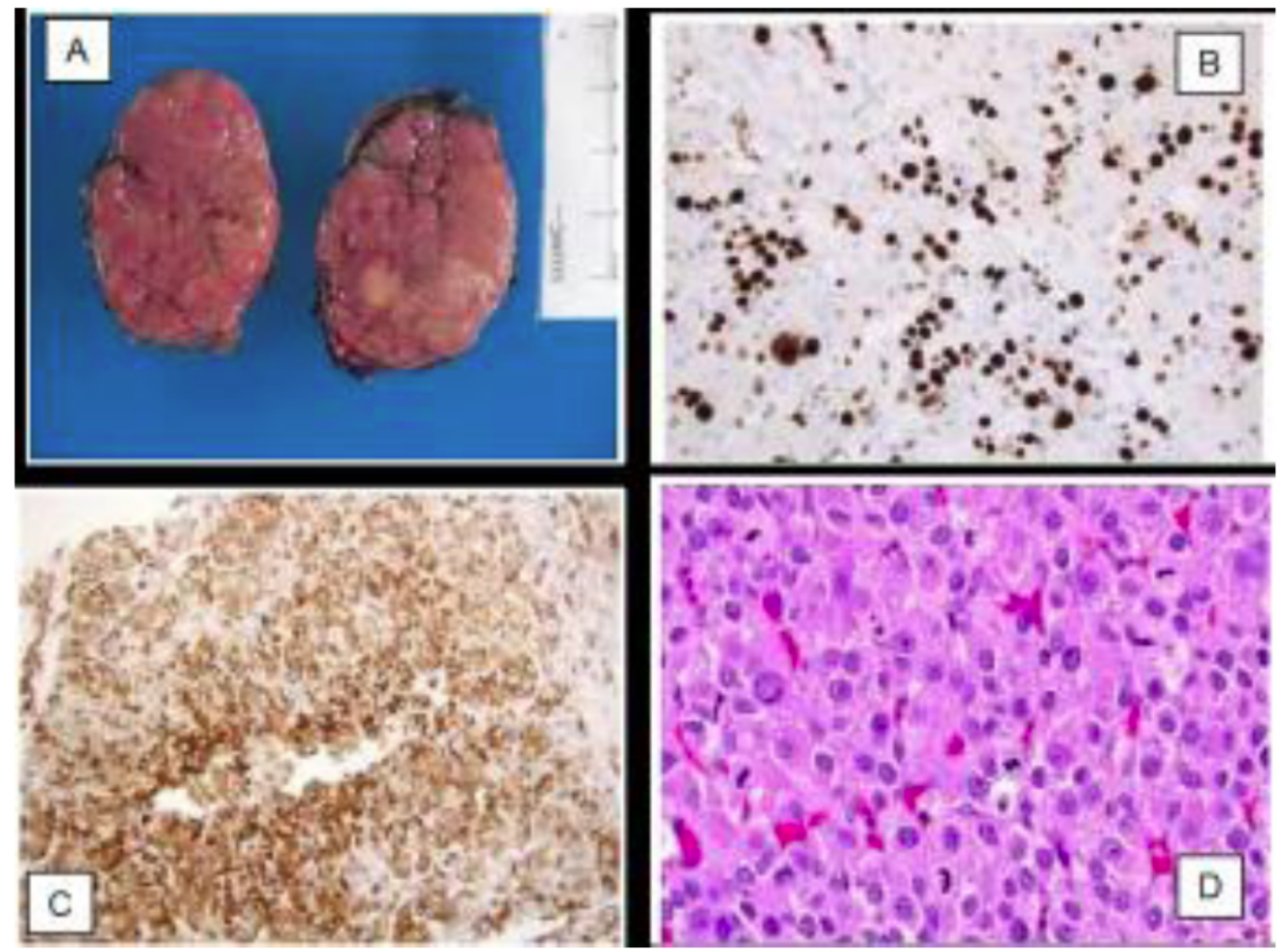

Figure 2. (A) Cut surface of tumor. (B) Ki-67 staining shows a high proliferative index. (C) Positive inhibin staining is typical of adrenal cortical neoplasm. (D) Section demonstrating multiple abnormal mitotic figures.

growth. Genetic analysis of CYP21A2 and CYP11B1 genes lacked known pathological mutations. A deleterious mutation $(\mathrm{R} 158 \mathrm{H})$ in TP53 region suggested Li-Fraumeni syndrome.

The patient received no chemotherapy because the tumor was well encapsulated and completely resected. Hormone levels were within normal levels at one week, 2 months, and 6 months after surgery. No tumor recurrence was observed on a postoperative abdominal ultrasound performed at 7 months.

\section{DISCUSSION}

Adrenocortical tumors in childhood are rare. They comprise fewer than $2 \%$ of all childhood tumors, with an incidence ranging from 0.30 to 0.38 per million [1]. These tumors have been associated with Beckwith-Wiedemann syndrome, hemihypertrophy syndrome, and Li-Fraumeni syndrome [2-4]. Overexpression of IGF-II and H19 genes in Beckwith-Wiedemann syndrome, or TP53 mutation in Li-Fraumeni syndrome have been proposed in the pathogenesis of adrenal carcinomas [2, 3]. High occurrence of ACT in association with TP53 mutation (especially, $\mathrm{R} 337 \mathrm{H}$ ) has been reported in Southern Brazil [3]. To our knowledge, our patient is the first report of
$\mathrm{R} 158 \mathrm{H}$ in TP53 associated with an ACT, suggesting Li-Fraumeni syndrome.

Although about $12 \%$ of cases with ACTs were reportedly diagnosed during the first year of life [4], few cases were diagnosed during the neonatal period $[4,5]$. Most neonatal ACTs were incidentally detected by the presence of suprarenal tumors on antenatal ultrasonography. Pediatric ACTs usually secrete hormones that cause virilization, precocious puberty, or Cushing syndrome [4, 5]. According to [5], based on the data of 254 patients, the median interval between the first symptoms and diagnosis was 5 months (range from 0 to 90 months). The diagnosis of ACT usually was delayed because of the generally healthy appearance of the child and the lack of a palpable abdominal mass. Our case lacked virilization, despite high levels of circulating androgens, including 17-OHP, testosterone, and DHEA. It is possible that this tumor occurred quickly in the last weeks of gestation before virilization could progress.

ACTs can also be misdiagnosed as $\mathrm{CAH}$, especially if 17-OHP is elevated. Screening newborns by measuring circulating $17-\mathrm{OHP}$ has been introduced in many countries worldwide for 
early detection of the most common form of $\mathrm{CAH}$ [6]. The diagnosis of $\mathrm{CAH}$ is confirmed by endocrinology and genotyping in selected cases, but confirmatory results are usually not immediately available.

Since elevated 17-OHP can be found in both $\mathrm{CAH}$ and androgen-secreting tumors, ACTs should be considered in the differential diagnosis of patients with elevated circulating androgen levels and a lack of expected virilization. Abdominal imaging studies are necessary when ACTs are suspected. Adrenal ultrasounds usually reveal an adrenal mass. Although inconsistently informative, adrenal ultrasound has been useful in the presumptive diagnosis and therapeutic management of $\mathrm{CAH}$ while awaiting confirmation by laboratory studies [7]. Symmetric enlargement of both adrenal glands is present in classic $\mathrm{CAH}$, in contrast to the asymmetric enlargement of one adrenal gland associated with an ACT [7]. Adrenal ultrasonography expedited early detection of the ACT reported here.

Distinguishing an adrenocortical adenoma from adrenocortical carcinoma on the basis of histologic findings is often difficult, especially in children. The Weiss scoring system might be of some value in predicting malignant behavior $[8,9]$. The prognosis of adrenal adenoma is excellent, in contrast to adrenocortical carcinoma [9].

Complete surgical resection of the ACT with replacement steroid therapy until the contralateral adrenal gland recovers is the preferred treatment. Because some ACTs secrete abnormally high levels of endogenous cortisol, the function of the contralateral adrenal gland may have become suppressed [10]. The benefits and risks of chemotherapy for ACTs in children remain controversial, including Mitotane or other chemotherapeutic agents [5].

In summary, ACTs should be considered in the differential diagnosis of neonates with elevated 17-OHP detected by newborn screening in the absence of expected virilization. Adrenal ultrasound is recommended during the initial evaluation of suspected adrenal neoplasms.

\section{ACKNOWLEDGEMENTS}

This work was supported by a Resident Faculty Mentoring Grant from the Department of Pediatrics, Loma Linda University School of Medicine.

\section{REFERENCES}

[1] Young JL Jr, Ries LG, Silverberg E, Horm JW, Miller RW. Cancer incidence, survival, and mortality for children younger than age 15 years. Cancer 1986; 58(2 Suppl): 598-602.

[2] Hoyme HE, Seaver LH, Jones KL, Procopio F, Crooks W, Feingold $M$. Isolated hemihyperplasia (hemihypertrophy): report of a prospective multicenter study of the incidence of neoplasia and review. Am J Med Genet 1998; 79: 274-8.

[3] Figueiredo BC, Sandrini R, Zambetti GP, et al. Penetrance of adrenocortical tumors associated with the germline TP53 R337H mutation. J Med Genet 2006; 43: 91-6.

[4] Sutter JA, Grimberg A. Adrenocortical tumors and hyperplasia in childhood-etiology, genetics, clinical presentation and therapy. Pediatr Endocrinol Rev 2006; 4: 32-9.

[5] Michalkiewicz E, Sandrini R, Figueiredo B, et al. Clinical and outcome characteristics of children with adrenocortical tumors: a report from the International Pediatric Adrenocortical Tumor Registry. J Clin Oncol 2004; 22: 838-45.

[6] Speiser PW, Azziz R, Baskin LS, et al. Congenital adrenal hyperplasia due to steroid 21-hydroxlase deficiency: an Endocrine Society clinical practice guideline. J Clin Endocrinol Metab 2010; 95: 41334160.

[7] Sivit CJ, Hung W, Taylor GA, Catena LM, BrownJones C, Kushner DC: Sonography in neonatal congenital adrenal hyperplasia. AJR Am J Roentgenol 1991; 156: 141-3.

[8] Weiss LM, Medeiros LJ, Vickery AL Jr. Pathologic features of prognostic significance in adrenocortical carcinoma. Am J Surg Pathol 1989; 13: 202-6.

[9] Sakoda A, Mushtaq I, Levitt G, Sebire N. Clinical and histopathological features of adrenocortical neoplasms in children: respective review from a single specialist center. J Pediatr Surg 2014; 49: 410-5.

[10] Sandrini R, Ribeiro RC, DeLacerda L. Childhood adrenocortical tumors. J Clin Endocrinol Metab 1997; 82: $2027-31$. 